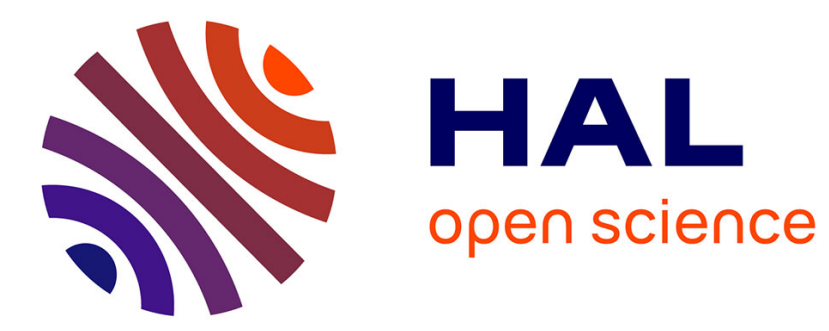

\title{
Sur les actions chimiques de la lumière (suite) \\ P. Villard
}

\section{To cite this version:}

P. Villard. Sur les actions chimiques de la lumière (suite). J. Phys. Theor. Appl., 1907, 6 (1), pp.445-457. 10.1051/jphystap:019070060044501 . jpa-00241226

\section{HAL Id: jpa-00241226 https://hal.science/jpa-00241226}

Submitted on 1 Jan 1907

HAL is a multi-disciplinary open access archive for the deposit and dissemination of scientific research documents, whether they are published or not. The documents may come from teaching and research institutions in France or abroad, or from public or private research centers.
L'archive ouverte pluridisciplinaire HAL, est destinée au dépôt et à la diffusion de documents scientifiques de niveau recherche, publiés ou non, émanant des établissements d'enseignement et de recherche français ou étrangers, des laboratoires publics ou privés. 
VILLARD. - ACTIONS GHIMIQUES DE LA LUMIĖRE 445

SUR LES AGTIONS CHIMIQUES DE LA LUMIÈRE (suite);

Par M. P. VILLARD (3).

PHÉNomènes de DESTRUCTION.

Les phénomènes de destruction, découverts par Ed. Becquerel, ont une généralité beaucoup plus grande que les effets continuateurs; ils n'exigent pas, comme ces derniers, la présence d'un sel d'argent soluble et peuvent, par suite, être observés avec toutes les préparations photographiques et même avec d'autres substances.

Le cas le plus simple et le plus frappant est celui du platinocyanure de baryum. On sait que ce sel, soumis à l'action des rayóns X,

(1) Kaufmann, Annalen der Physik, 19, p. $549 ; 1906$.

(2) Guye, Archives de Genève; mars et avril 1906.

(3) Voir ce volume, p. 369.

J. de Phys., $4^{\text {e }}$ série, t. VI. (Juin 1907.) 
brunit progressivement et perd en grande partie ses propriétés fluorescentes; or il suffit de l'exposer ensuite à la lumière pour faire disparaître la teinte brune et rétablir le sel dans son état primitif; l'expérience peut être répétée un grand nombre de fois avec le même écran et donne constamment le même résultat. Quelle que soit la transformation physique ou chimique qui s'est produite, il est manifeste que l'action de la lumière a été exactement inverse de celle des rayons $\mathrm{X}$.

Les radiations lumineuses qui produisent la régénération du platinocyanure de baryum forment dans le spectre trois groupes distincts situés respectivement dans le vert, le jaune et le rouge.

$\mathrm{Si}$ on renversait l'ordre de l'expérience, la lumière agissant la première ne produirait rien, et les rayons $\mathrm{X}$ venant après bruniraient le sel. Les rayons $\mathrm{X}$ et la lumière ne sont pas interchangeables.

Dans cette expérience, comme dans tous les phénomènes de destruction, le retour à l'état initial est d'autant plus rapide, ou exige une quantité de radiations d'autant moins grande que l'impression primitive était plus faible.

On obtient des résultats tout à fait analogues en prenant une plaque photographique (plaque Jougla) au gélatino-bromure d'argent et la traitant successivement par les rayons $\mathrm{X}$ et par la lumière. Le phénomène est toutefois moins simple si on emploie la lumière blanche, attendu que celle-ci peut impressionner la plaque sans le concours préalable des rayons $\mathrm{X}$, ce qui n'avait pas lieu pour le platinocyanure de baryum. Néanmoins, si la plaque a été exposée pendant une à deux minutes à des rayons $\mathrm{X}$ intenses (pose relativement faible, car il faudrait dix à quinze minutes pour obtenir un commencement de solarisation), puis traitée par la lumière (une minute à 40 centimètres d'un bec Auer), on constate que cette plaque est redevenue capable de donner une radiographie négative ordinaire, avec un temps de pose peu différent de celui qu'exigerait une plaque non traitée. Le gélatino-bromure voilé par les rayons $\mathrm{X}$ a donc été ramené par la lumière à un état qui, pour les rayons $\mathrm{X}$, est peu différent de l'état initial. Bien entendu, la plaque restaurée pour les rayons $\mathrm{X}$ ne l'est pas pour la lumière et ne peut plus donner une photographie.

$\mathrm{Si}$ on inverse l'ordre dans lequel on fait agir les radiations, la destruction n'a plus lieu; les rayons $\mathrm{X}$ ajoutent leur action à celle de la 
lumière, mais ne la détruisent pas; avec les temps de pose convenant à l'expérience précédente, on aurait non pas une bonne radiographie, mais une épreuve entièrement noire.

Pour analyser le phénomène, il convient de prendre une série de plaques semblables qu'on expose respectivement aux rayons $\mathrm{X}$ pendant des temps croissant depuis zéro jusqu'à cinq minutes, et qu'on traite toutes ensemble par une même lumière croissant d'un bord à l'autre de la plaque de zéro à cinq minutes, par exemple à วั0 centimètres d'un bec Auer. Les résultats du développement sont les suivants :

Pour la première plaque (pas de rayons X), le noircissement part de zéro, croît jusqu'à un maximum $M$ (pour une minute de pose environ), puis redescend par effet de solarisation.

Sur les plaques suivantes, le noircissement ne part pas de zéro; sur le bord non exposé à la lumière il a la valeur qui correspond à l'action des rayons $\mathrm{X}$ seuls, je l'appellerai le noir initial. En avançant vers le côté le plus éclairé de la plaque, le noircissement augmente encore, mais le maximum est atteint plus tôt, et il est inférieur à M. La descente ultérieure est ensuite plus accentuée qu'en l'absence des rayons $\mathrm{X}$.

A mesure qu'on avance dans la série des plaques, le noir initial augmente, le maximum est atteint de plus en plus vite et baisse de plus en plus.

Pour une certaine exposition aux rayons $\mathrm{X}$ (une minute environ), le maximum se confond avec le noir initial; le noircissement ne fait ensuite que descendre sous l'action de la lumière et tombe presque à zéro. Pour cette pose (pose stricte), toute exposition à la lumière, si courte qu'elle soit, ne fait qu'amoindrir le noircissement. Si on dépasse un peu cette exposition stricte aux rayons $\mathrm{X}$, les résultats sontencore bien plus nets, le noir initial est plus intense, et une plaque ainsi traitée, puis utilisée dans un appareil photographique ordinaire et développée par les méthodes usuelles ( $\left.{ }^{1}\right)$, fournit une image positive directe détaillée et vigoureuse. Dans cette image, les noirs sont produits par les rayons $\mathrm{X}$ et les blancs par la lumière; si l'exposition aux rayons $\mathrm{X}$ a dépassé la pose stricte indiquée plus haut, toutes les parties de l'image seront positives, car, si faible qu'ait été l'action de la lumière (détails dans les grandes ombres du sujet), cette action aura éclairci

(1) L'hydroquinone paraît préférable pour obtenir des noirs intenses. 
le noir dû aux rayons $\mathrm{X}$. Les blancs s'obtiendront très purs, presque aussi transparents que ceux d'un positif pour projection, si l'exposition à la lumière a été assez longue pour que l'impression produite par les rayons $\mathrm{X}$ soit totalement détruite dans les plages représentant, par exemple, des nuages blancs, des glaciers ou simplement le ciel. Comme on le verra plus loin, toutes les radiations spectrales sont actives dans cette expérience, et l'épreuve est à peu près orthochromatique.

L'exposition à la lumière doit toutefois être très longue, ce qui exclut la photographie instantanée. Dans les conditions où on aurait, par ce procédé ordinaire, une bonne épreuve en $\frac{1}{10}$ de seconde, il faut poser au moins une minute, c'est-à-dire 600 fois plus.

Supposons enfin qu'on dépasse beaucoup la pose stricte et qu'au lieu de deux minutes d'exposition aux rayons $\mathrm{X}$ on arrive à quinze minutes ou vingt minutes, il y a un commencement de solarisation de la plaque; ce que j'ai appelé le noir initial est moindre que pour des expositions plus courtes; mais l'action affaiblissante de la lumière reste la même.

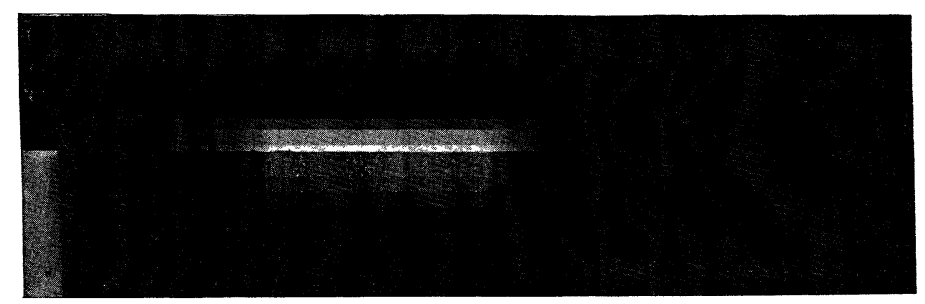

FIG. 1. - Positifs directs obtenus l'un avec le concours des rayons X, l'autre par simple solarisation.

Spectre solaire photographié sur plaque Jougla dont la partie supérieure a été seule exposée aux rayons $\mathrm{X}$ ( 3 minutes).

Temps de pose (commun aux deux images) dans le spectrographe : 4 minutes.

Le voile de la partie inférieure de la plaque est dù à la lumière diffusée par le système optique pendant la pose.

On voit à gauche les raies $\mathrm{H}$ et $\mathrm{K}$. A droite, le spectre supérieur se termine à $\mathbf{A}$ (difficilement visible sur le cliché original).

Ces effets, évidemment complexes dans le cas de la lumière blanche, ressemblent à la solarisation. Ce dernier phénomène étant inexpliqué, on ne peut savoir s'il n'existe rien de commun entre lui 
et ce qu'on observe dans les expériences précédentes, mais on peut affirmer qu'il n'y a pas identité : une plaque voilée par la lumière jusqu'au début de la solarisation, puis exposée dans un appareil photographique, donnerait bien un positif, mais l'image serait grise et elle ne serait positive dans toutes ses parties que si le voile préalable a atteint le commencement de la solarisation. Avec les rayons X, ce voile en est fort loin, la meilleure exposition étant le dixième environ de ce qu'il faudrait pour qu'il en fût ainsi.

En second lieu, il serait indifférent de voiler la plaque avant ou après son passage dans la chambre photographique, la quantité totale de lumière reçue par une région de la plaque étant évidemment la même dans les deux cas. Or, si on essaye de faire agir les rayons $\mathrm{X}$ après l'exposition derrière l'objectif, on obtient, au lieu d'une bonne épreuve, un voile intense presque uniforme. L'hypothèse d'un effet de solarisation ne rendrait pas non plus compte de ce fait que la plaque, traitée par les rayons $\mathrm{X}$, puis par la lumière, redevient capable de s'impressionner par ces rayons, cette nouvelle impression pouvant encore être effacée par la lumière, tout comme la première.

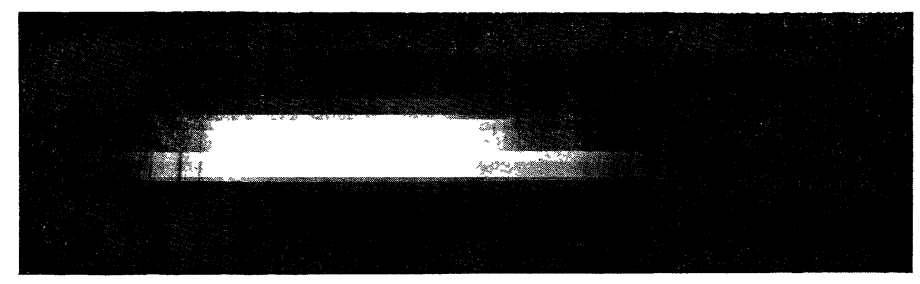

Fig. 2. - Epreuve représentant indifféremment le résultat de l'une ou l'autre des expériences suivantes, une seule reproduction ayant été faite pour cause d'identité :

$1^{\circ}$ Plaque Jougla traitée par les rayons X (4 minutes).

Exposition au spectre en trois poses : 20 secondes, 2 minutes, 10 minutes. $2^{\circ}$ Traitement par les rayons $\mathrm{X}$ ( 4 minutes).

Exposition à la lumière d'une lampe Nernst: 30 secondes.

Nouveau traitement par les rayons X : 4 minutes.

Exposition au spectre (trois poses) : 20 secondes, 2 minutes, 10 minutes.

Enfin, une différence fondamentale est fournie par ce fait que plus l'exposition aux rayons $\mathrm{X}$ a été longue (pourvu qu'elle soit inférieure à 15 minutes), plus il faut de lumière pour revenir au blanc. Avec la solarisation, ce serait exactement le contraire : plus on se 
serait approché de la pose solarisante, moins il resterait à faire pour l'atteindre.

Ces phénomènes, joints à l'impossibilité de renverser l'ordre des choses sans transformer complètement les résultats, montrent qu'il y a dans l'action de la lumière agissant après les rayons $\mathrm{X}$ quelque chose d'entièrement dífférent des effets photographiques ordinaires.

L'emploi d'un spectroscope photographique permet d'analyser ces phénomènes de destruction.

Dans la région du maximum de sensibilité de la plaque (vers $G$ solaire), l'effet produit sur la plaque röntgénisée est le même qu'avec la lumière blanche. Plus la dose de rayons X est forte, plus il faut de lumière pour détruire leur action; mais un minimum de rayons $\mathrm{X}$ est nécessaire pour que les effets destructeurs apparaissent dans toute leur netteté. Cette complication disparaît à mesure qu'on s'éloigne du maximum de sensibilité de la plaque.

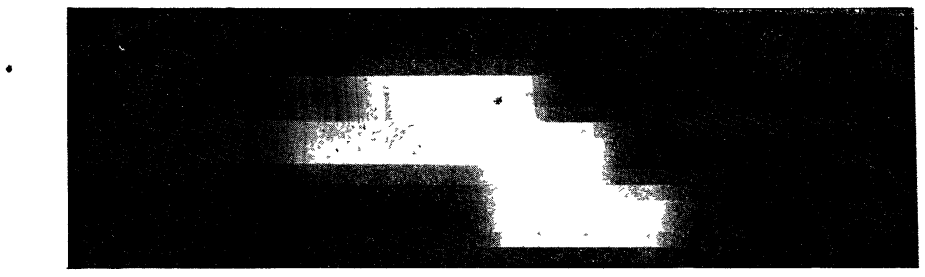

Fig. 3. - Expérience montrant que plus l'impression produite par les rayons $\mathbf{X}$ est faible, plus le retour au blanc est facile.

La partie supérieure de la plaque (Jougla) a été traitée par.les rayons $X$ pendant 4 minutes, la partie inférieure pendant 1 minute.

Exposition commune dans le spectrographe : 4 minutes (résultat analogue de 1 minute à 10 minutes).

Dans le violet extrême, on peut se contenter d'une moindre exposition aux rayons $X$ et on obtient plus aisément des blancs parfaits. Dans l'extrême rouge et l'infra-rouge( $\left.{ }^{1}\right)$, les résultats deviennent d'une simplicité absolue. La plus faible dose de rayons $\mathrm{X}$ capable d'être décelée par le révélateur suffit pour observer le phénomène, et le retour au blanc est d'autant plus rapide que le voile préalable était moindre. Dans ces conditions, la photographie de l'extrême

(1) Il ne faut pas employer les plaques à l'iodobromure d'argent, qui sont sensibles au rouge extrême du spectre. 
rouge (au delà de $\mathrm{B}$ solaire) et de l'infra-rouge jusqu'à $1 \mu$ environ (prisme de flint) peut se faire avec le temps de pose qui, pour le même spectre, donnerait, en photographie ordinaire, un bon négatif dans le violet extrême, vers $\mathrm{K}$ solaire. On est alors singulièrement loin des poses qui avoisinent la solarisation.

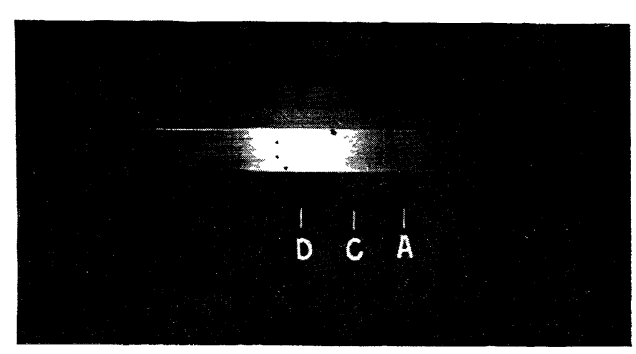

F1a. 4. - Partie peu réfrangible du spectre; exposition aux rayons $\mathbf{X}$ (1 minute).

Exposition dans le spectrographe, derrière un verre jaune, 30 secondes pour la partie supérieure du spectre, 10 minutes pour la partie inférieure.

(Ces temps de pose peuvent être réduits de moitié pour un cliché non destiné à la reproduction en similigravure.)

Les raies du spectre sont indiquées au-dessous de l'image. La comparaison avec les figures précédentes montre qu'en réduisant l'exposition aux rayons $\mathbf{X}$ on réduit aussi le temps de pose nécessaire à l'obtention du positif; la raie $A$, très visible ici, n'était pas atteinte dans les figures 2 et 3 .

Les propriétés destructives de l'infra-rouge permettent de détruire l'impression par les rayons $\mathrm{X}$, même au travers de trois feuilles de fort papier noir. La lumière d'un bec Auer suffit pour cela, une exposition de quelques heures étant toutefois nécessaire, vu l'opacité du papier pour les radiations même infra-rouges. Dans les mêmes conditions, une plaque non traitée ne donne naturellement rien $(f g .5)$.

On réalise des effets analogues en voilant une plaque quelconque par la lumière ordinaire et la traitant ensuite par le rouge extrême et l'infra-rouge. On retrouve alors, sur le gélatino-bromure ou chlorure d'argent, les résultats obtenus par Ed. Becquerel sur d'autres préparations. Plus le voile préalable est faible, moins il faut de lumière rouge pour l'effacer. Ces expériences se font sans difficulté dans un spectroscope; il convient seulement, à cause de la diffusion notable produite par les lentilles et les prismes, d'atténuer le violet et le bleu au moyen d'un verre jaune. Avec une lampe Nernst de 60 bougies et une fente de 1 à $\mathscr{L}$ millimètres, dix à quinze minutes 
suffisent pour effacer, sur gélatino-bromure, un voile un peu infé-

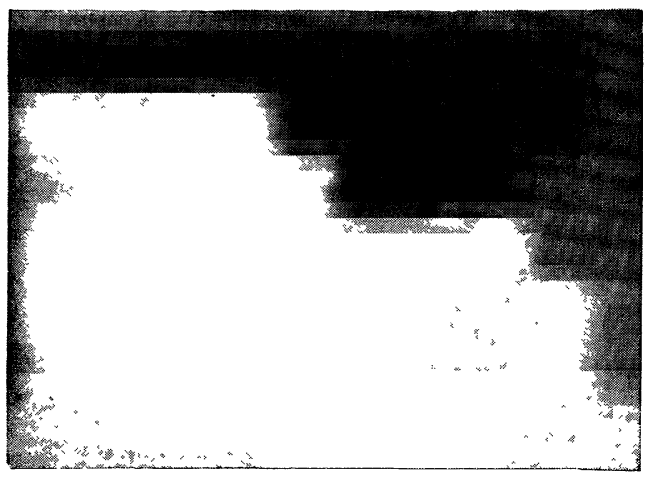

Fig. 5. - Expérience de destruction au travers de trois feuilles de fort papier noir.

Ombre d'une roue d'engrenage projetée par un bec Auer sur plaque Jougla dont la partie supérieure a été exposée 30 secondes aux rayons $\mathbf{X}$.

Durée de l'exposition devant le bec Auer : 2 heures. Aucune trace d'impression sur la partie non traitée de la plaque.

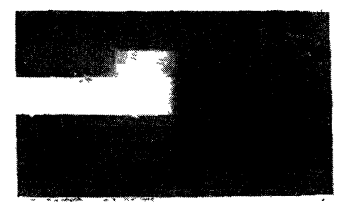

Fig. 6. - Expérience montrant que l'ordre dans lequel on fait agir les radiations n'est pas indifférent.

Le tiers supérieur de la plaque (gélatino-chlorure d'argent) a été exposé aux rayons rouges après voile par la lumière ordinaire, la partie inférieure avant un voile identique. Le tiers moyen n'a pas été voilé. Les temps de pose respectifs sont les mêmes pour les deux groupes de radiations employées.

Nota. - Autour de l'épreuve a été reproduit un fond blanc pour montrer que la similigravure ne peut traduire le blanc pur que par du gris qui est l'image de la trame. Cette remarque est, par suite, applicable à toutes les figures de cet article et de l'article précédent.

rieur à la pose photographique normale que tout le monde sait 
apprécier. Pour le gélatino-chlorure, une heure environ est nécessaire $\left.{ }^{1}\right)$.

$\mathrm{Si}$ on inverse l'ordre dans lequel on fait agir les radiations, c'est-à-dire si on fait succéder le bleu ou le violet au rouge, tout se passe comme si la plaque n'avait pas été exposée à la lumière rouge $(f g .6)$.

Le traitement par la lumière rouge ramène la plaque à son état initial.

L'emploi du gélatino-chlorure dit pour tons chauds, presque insensible au jaune, permet de le vérifier même sans spectroscope. On

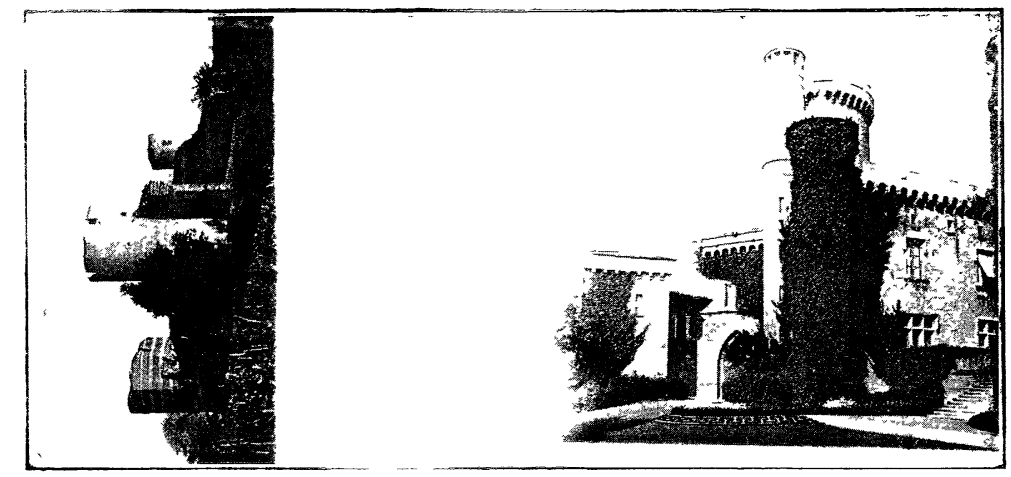

Fig. 7. - Destruction d'uıc inàge latente et restauration de la sensibilité par le rouge.

Une plaque Perron (gélatino-chlorure pour tons chauds) a été exposée sous un cliché stéréoscopique pendant le temps normal.

L'épreuve de droite a été conservée comme témoin, l'épreuve de gauche effacée par exposition au soleil sous des verres jaunes.

Sur une partie de cette région de la plaque, une nouvelle image a été imprimée avec le temps de pose normal. Le tout a été ensuite développé.

Le dégradé du bord gauche de l'épreuve de droite est dû à la diffusion des rayons destructeurs dans l'émulsion et représente les phases successives de la destruction.

peut par exemple faire sur une plaque de ce genre une épreuve stéréoscopique; puis, l'une des images étant conservée comme témoin, l'autre est, avant développement bien entendu, exposée au soleil pendant quatre heures sous des verres jaunes bien choisis. Au bout

(1) Pour ces expériences, le gélatino-chlorure d'argent est plus commode que le gélatino-bromure; il est à peine sensible au jaune et au vert, et il suffit de mettre un verre jaune devant la fente pour éviter toute impression parasite gênante. Avec le gélatino-bromure, il faut arrêter le vert et même le jaune. 
de ce temps, la plaque est apte à recevoir une nouvelle image, et sa sensibilité est presque rigoureusement la même qu'avant l'expérience, comme on peut aisément s'en assurer par un essai comparatif fait avec une autre plaque de la même boîte.

Les phénomènes de protection, déjà signalés par Ed. Becquerel et qui ne sont qu'une variante des précédents, s'observent sans difficulté dans le spectroscope photographique. Il suffit de laisser une plaque exposée dans l'appareil pendant un temps quelconque. L'inévitable lumière diffusée par le système optique, et qu'il faut même atténuer par un verre jaune faible, voile plus ou moins, suivant la durée de l'expérience, toute la surface de la plaque; mais, dans la région occupée par le rouge et l'infra-rouge, la préparation reste intacte, les rayons destructeurs effaçant le voile à mesure qu'il se produit.

Le succès de l'expérience dépend non du temps de pose, mais de la proportion réalisée entre les deux radiations antagonistes.

Les papiers pour noircissement direct se prêtent aussi à ces expériences; si on les développe, on rentre dans le cas des plaques, et il est plus intéressant de ne pas faire intervenir le révélateur ; on constate alors par exemple les effets suivants: si le voile produit par la lumière est assez faible pour être juste visible, la lumière rouge le fait disparaître par blanchissement direct ( $\left.{ }^{1}\right)$. L'expérience est facile à faire avec un bon verre rouge doublé d'un verre jaune pour arrêter le bleu que laissent passer presque tous les verres rouges, ou mieux avec un filtre à sélénium vitreux $\left({ }^{2}\right)$. Le spectroscope convient également, mais la durée de l'expérience devient inacceptable.

Une image faible ainsi visiblement effacée ne donne pas prise aux révélateurs physiques ou chimiques (acide gallique et azotate d'argent, ou hydroquinone) ni aux rayons continuateurs, elle est réellement supprimée.

Ce blanchissement direct ne se produit pas avec du chlorure d'argent seul.

(1) Pour les papiers à noircissement direct, le développement n'existant pas, ou plus exactement se faisant à mesure que la lumière agit, la question de retour au blanc par solarisation ne se pose évidemment pas. On n'a d'ailleurs jamais vu du papier sensible blanchir par excès d'insolation.

(2) On fond un fragment de sélénium sur une lame de mica, et on l'écrase rapidement avec une autre lame pressée par un corps froid; ce filtre arrête tout ce qui est plus réfrangible que $G$ solaire. Une lampe à mercure regardée au travers du sélénium parait rouge et la supériorité sur le verre rouge apparaît immédiatement. 
L'action destructive du rouge se manifeste plus nettement lorsqu'on essaye de continuer une image faible en la traitant par un mélange de rouge et de jaune. Il convient d'opérer par comparaison en prenant un papier qu'on impressionne sous un cliché stéréoscopique jusqu'à commencement de visibilité des grands noirs. Le cliché étant alors supprimé, une des deux images est exposée au soleil sous verres jaunes et verts, l'autre sous verres jaunes et verre rouge faible. Par un choix convenable de ces filtres, on arrive aisément à ce que, dans les deux images, les noirs montent avec la même vitesse. L'expérience terminée, on constate que sous les verres jaunes et verts tous les détails sont venus, tandis que l'épreuve exposée aux rayons jaunes et rouges, bien qu'aussi intense dans les noirs, est heurtée et sans détails dans les blancs (effet de neige), c'est-à-dire dans les parties qui correspondaient aux fortes opacités du cliché. Autrement dit, toutes les impressions faibles ont disparu au lieu de se développer par continuation. L'explication de ce fait est fort simple : la destruction est d'autant plus rapide que l'impression à détruire est plus faible; l'inverse a lieu pour la continuation. 11 résulte de là que les grands noirs de l'image (impressions fortes) seront à peine attaqués par le rouge pendant le temps nécessaire à leur renforcement complet; au contraire, les impressions faibles auront été effacées bien avant que les rayons continuateurs n'aient pu les développer d'une manière appréciable (1).

Les effets de protection s'observent sans peine avec les papiers à noircissement direct.

Il suffit de recevoir un spectre sur une feuille sensible (chlorure et azotate d'argent additionné d'acide citrique ou tartrique pour en assurer la conservation). Le spectre s'imprime d'abord, limité à

(1) Dans cette expérience et dans la plupart des autres, l'usage de véritables images photographiques se montre infiniment supérieur à la méthode des expositions par échelons que j'avais employée en 1898 à propos des rayons $\mathbf{X}$, et qui semble au premier abord plus géométrique. Avec cette méthode on laisse échapper une foule de phénomènes qui apparaissent très franchement sur une image. D'insignifiantes différences font que l'épreuve est voilée ou manque de soleil, produisent les effets dits de neige ou de charbon, et se manifestent ainsi à première vue sans qu'il y ait lieu de faire une mesure photométrique. La raison en est que les impressions variées qui produisent l'image, bien que n'étant pas classées par ordre, ne sont pas pour cela distribuées au hasard; elles figurent des objets parfaitement connus, et le moindre défaut de ressemblance, inappréciable aux mesures, apparaît immédiatement, et l'interprétation n'en est jamais difficile. 


\section{VILLARD. - ACTIONS GHIMIQUES DE LA LUMIÈRE}

l'orangé, puis la lumière diffusée voile peu à peu tout le reste de la feuille. Si cette lumière diffusée n'est pas trop intense, on observe que la région rouge et infra-rouge du spectre reste indéfiniment blanche et se détache de plus en plus sur le fond qui s'assombrit progressivement. On conçoit que la proportion de rayons actiniques, que la diffusion par les prismes a mélangés au rouge, ne doit pas dépasser une certaine valeur: on est dans de bonnes conditions avec une forte lampe Nernst ; aucun verre absorbant n'est alors nécessaire. La $f g .8$ montre le résultat obtenu avec une exposition de trente jours $\left({ }^{1}\right)$.

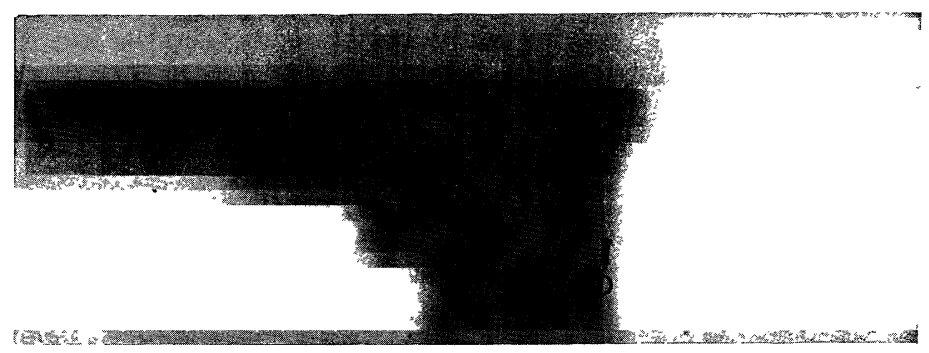

Fig. 8. - Effet de protection produit par les rayons destructeurs sur un papier pour noircissement laissé pendant trente jours dans un spectrographe éclairé par une lampe Nernst (pas de développement).

Ainsi une radiation convenablement choisie peut détruire l'impression produite par une autre, et l'inverse n'a pas lieu $\left({ }^{2}\right)$. Les rayons destructeurs ne sont pas les mêmes pour toutes les substances sensibles : pour le platinocyanure de baryum, par exemple, ils sont autres que pour le chlorure d'argent. Enfin la destruction est d'autant plus rapide que l'impression à détruire est moindre, et la sensibilité est généralement restaurée.

(1) Les rayons solaires, filtrés par de bons verres rouges et jaunes, protègent également le papier contre l'altération spontanée qu'il subit d'ordinaire avec le temps, même dans une boîte bien close.

(2) Une comparaison donnera une image exacte de ce phénomène; on peut avec un grattoir effacer une tache d'encre sur une feuille de papier; mais il est évident que le résultat ne sera pas le même si on inverse l'ordre des opérations. 


\section{APPENDICE.}

$1 l$ m'a paru intéressant pour les lecteurs du Journal de Physique de reproduire les quelques lignes dans lesquelles Ed. Becquere! résume ses travaux sur les rayons continuateurs et destructeurs ( $l a$ Lumière, ses causes, ses effets, Paris, Firmin-Didot, 1868, p. 94):

“ En résumé.... on reconnaît dans l'action du rayonnement les trois effets suivants :

“ $\mathbf{1}^{\circ}$ Effet produit dans le spectre solaire depuis le bleu jusqu'au delà du violet sur tous les papiers, verres colledionnés ou lames d'argent recouvertes d'iodure, de chlorure ou de bromure de ce métal, et, dans le cas de la lame d'argent bromée, effet pouvant aller, du côté le moins refrangible, jusqu'à l'orangé; les limites d'action ont été indiquées plus haut;

" $2^{\circ}$ Effet de continuation sur les papiers et les surfaces enduites d'iodure, de chlorure ou de bromure avec excès d'azotate d'argent, ainsi que sur les plaques iodées, puis chlorées, depuis la raie B dans le rouge jusque près du bleu, lorsque ces surfaces ont été préalablement impressionnées. Sur les plaques d'argent iodées et bromées, on n'observe aucun effet de ce genre, ainsi que sur le chlorure et le bromure d'argent précipités isolément et lavés ;

" $3^{\circ}$ Effet des rayons rouges extrêmes et infra-rouges pour rendre les surfaces d'argent iodées et iodées-bromées, préalablement insolées, incapables de condenser la vapeur de mercure et les placer dans des conditions semblables à celles où elles étaient avant l'insolation. Cet effet est analogue à celui que l'on observe lorsque ces mêmes rayons rouges extrêmes retardent ou s'opposent à la coloration des papiers sensitifs à la lumière diffuse. ” 